\title{
A review of fatal accident incidence rate trends
}

\section{in fishing}

\author{
Olaf C. Jensen ${ }^{1}$, Gudrun Petursdottir ${ }^{2}$, Ingunn Marie Holmen ${ }^{3}$, \\ Annbjørg Abrahamsen ${ }^{4}$, Jennifer Lincoln ${ }^{5}$ \\ ${ }^{1}$ Centre of Maritime Health and Society, Institute of Public Health, University of Southern Denmark, Esbjerg, Denmark \\ ${ }^{2}$ Institute for Sustainability University of Iceland, Reykjavik, Iceland \\ ${ }^{3}$ SINTEF Fiskeri og havbruk, Trondheim, Norway \\ ${ }^{4}$ Department for Occupational Medicine and Public Health, The Faroe Islands Health System, The Faroe Islands \\ ${ }^{5}$ The National Institute for Occupational Safety and Health, Alaska Pacific Office, United States
}

\begin{abstract}
Background: Injury prevention in fishing is one of the most important occupational health challenges.

Aim: The aim was to describe and compare internationally the trends of the fatal injury incidence rates and to discuss the impact of the implemented safety programs.

Materials and methods: The review is based on journal articles and reports from the maritime authorities in Poland, United Kingdom, Norway, Iceland, Denmark, United States and Alaska and Canada. The original incidence rates were recalculated as per 1,000 person-years for international comparison of the trends. Results: The risk of fatal accidents in fishing in the northern countries has been reduced by around $50 \%$ to an average of about 1 per 1,000 person-years. Norway and Canada keep the lowest rates with around 0.5 and 0.25 per 1,000 person-years. About half of the fatal injuries are related to vessel disasters and drowning. The safety programs seem to have good effects, but the risk is still about 25 to 50 times higher than for onshore workers.

Conclusions: The overall fatal injury rates in the European and North American studies decreased by around $50 \%$ most probably as result of the implemented safety programs. However the high risk in fishing compared to onshore workers calls for continued and intensified safety programs.
\end{abstract}

(Int Marit Health 2014; 64, 2: 47-52)

Key words: fishing, occupational, fatal, injury, epidemiology, incidence, fatigue

\section{INTRODUCTION}

It is generally agreed that an adequate method to prevent fishing injuries and vessel disasters is to adapt the risk management and risk prevention systems used successfully by other industries. Of course, the specific conditions in fishing must be taken in consideration. The cornerstone of risk prevention is the risk assessment process which is carried out as a stepwise process, starting with identification of the hazards and the persons at risk [1]. Here we focus on the overall, epidemiological part of the documentation for the risk assessment process, especially for fatal injuries that will also be relevant for prevention of non-fatal injuries.
One important characteristic of the historical development of the fishing fleet structure is the diversity of type and size of fishing vessels to be taken in consideration in the prevention plans. At one end of the spectrum are the multinational companies utilising large factory trawlers, and at the other end, in developing countries, are the small, wooden canoes and other boats used to catch sufficient food for the local communities. For many years, the industry has been economically and socially depressed due to significant declines in stocks of fish. The fishing quotas regulations have grown more intense over the years with strong financial impact and most possibly negative impact 
on the safety standards. Three hundred years ago, fishing was described as one of the most extreme work conditions [2]. About 60 years ago fishing in the United Kingdom was described as one of the most extreme occupations [3]. Recently Conway [4] described fishermen as "Casting their lot upon the water". In 1976-1995, the United Kingdom fishing fatality rate ranged on top of all industries, and was the double of merchant seafaring [5]. Today, injury prevention in fishing is still a most important challenge globally. The objectives are: (1) to describe the fatal injury rate trends of the latest decades; (2) to point out the main causal factors and (3) to discuss the impact of the implemented prevention programs.

\section{MATERIALS AND METHODS}

We applied the epidemiological method to describe the fatal incidence rates based on the information from peer-reviewed journal articles and the national Maritime Authority reports. The observation period included the latest decades but also some earlier data were included. The Medline database and the Google were searched with the keywords "injuries", "epidemiology", "incidence", "accident", "fishing", and "fisherman." Only articles and administrative reports with precise denominator and nominator data were included. Further the references served as supplemental source of information. Commercial fishermen in Norway, Iceland, Denmark, United Kingdom, Poland, Canada, United States and Alaska were selected for the review study as they are supposed to be more or less economical and technical comparable in the cold climate of the northern part of the globe. The terms "accident" and "injury" are used synonymously for the same type of harmful event. An occupational injury has been defined as a sudden and unexpected harmful event at work. The precise numbers of fatalities and the numbers of fishermen's populations per year were extracted from the articles and the reports and the incidence rates were adjusted in Excel for comparison of the incidence rate to fatalities per 1,000 fishermen per year or full time equivalent fishermen per year. For example, from 1980 to 1984 there was on average 25 fatal accidents per year per 10,000 Icelandic fishermen [6]. By dividing the numerator and the denominator with 10 the incidence rate is 2.5 per 1,000 fishermen per year. The rates cannot be compared directly but we can follow the increase and decrease of the development of the rates in parallel over the years. The information on the direct and indirect causes of the accidents was extracted from the articles and the reports. The trends and the main causal risk factors are presented for each country based on the information given in the articles and the reports. The impact of the implemented safety programs over the years on the fatal injury rate trends is evaluated in the discussion.

\section{RESULTS}

\section{TRENDS OF THE FATAL INJURY RATES}

The fatal injury rates in Norwegian fishing decreased from 1955 to 2006, except for the two periods of 1980-1984 and 1990-1994 due total losses of larger vessels $[7,8]$. The incidence rates are calculated per "man-labour years", based on the precise number of days at sea for every fisherman. The incidence rates (calculated) declined from 1.0 to 0.7 per 1,000 man-labour years during these years. For the period 1998-2006, the calculated risks were respectively $2.5,0.6$, and 0.2 fatal injuries per 1,000 man-labour years for small coastal vessels (Loa) $<13 \mathrm{~m}$, medium coastal vessels $13-28 \mathrm{~m}$, and deep sea fishing vessels $>28 \mathrm{~m}$.

The fatal rate in Icelandic fishing was $0.89 / 1,000$ in 1966-1986 and continued to decline from 1980 to 2005 to a level below 0.5 per 1,000 man-years [6]. The study population was "Icelandic seafarers" that also included seafarers from the merchant fleet who add to the lower injury rate. Another explanation of the low rate is that they mainly use large fishing vessels with a higher safety standard than for the smaller vessels [9].

The fatality rates in Danish fishing decreased from 2.4 in 1970-1972 to 1.36 for 1988-1992 per 1,000 person-years $[10,11]$. For 1995-2005, the overall rate was 1 per 1,000 fishermen per year and nearly stable during that period [12]. Among the fatalities in Danish fishermen in 1989-2005, 53\% occurred in 36 vessel disasters. The rate of disasters among the Danish fishing ships continued with a slight decreasing slope during 2000-2009 and the rate of fatal accidents remained decreased to around 1 or less per 1,000 registered fishermen [13].

For the United Kingdom in the period 1992-2006 the average fatality rate was 1.26 deaths per 1,000 fishermen per year, but with a fair degree of fluctuation. There is only a very small reduction over the 15 years in the rate of fatalities [14]. The fatal rate for United Kingdom fishermen for 1996-2005 was 115 times higher than that of the general workforce in Great Britain. While the fatal accident rate for almost all other United Kingdom occupations fell sharply over the last 30 years, there was no discernible reduction in the fishing industry [5].

In the Polish small-scale fishing industry a total of 177 deaths at sea was identified between the years 1960-1999 and $96 \%$ of these were due to external (non-medical) causes [15]. The calculated overall injury rate was 0.9 deaths per 1,000 fishermen per year. The highest incidence rates were in the smaller boats $<13 \mathrm{~m}$ in length.

Among commercial fishermen in the Canadian Atlantic coast during 1975-1983 there were 84 fatal fishing accidents in 183,378 person-years resulting in a fatal injury 
rate of 0.45 per 1,000 fishermen per year [16]. According to the authors, the study is likely to underestimate the risk due to a supposed underreporting. During 1999-2010 a total of 154 fatalities were reported from the Canadian commercial fishing among a total of 650,233 person-years with an average cumulative incidence rate of 0.24 per 1,000 fishermen per year. The variation is from 0.30 to 0.15 fatalities per 1,000 fishermen/year with an overall decreasing trend [14].

The number of commercial fishing deaths in the United States has been declining gradually since 1992. In the 1990 s, the number of deaths per year ranged from 48 to 96 compared to 41 to 61 deaths occurred annually during 2000-2009 [17]. During 2000-2010, an annual average of 46 deaths occurred (1.24 deaths per 1,000 workers), compared with an average of 0.04 per 1,000 workers among all United States workers. The most hazardous fisheries in the United States based on fatality rates from 2000 to 2009 are: Northeast multispecies ground fisheries, 6 fatalities per 1,000 full time equivalent workers (FTEs) and Atlantic scallop fishery, 4.3 fatalities per 1,000 FTEs, West Coast Dungeness crab fishery, 3.1 fatalities per 1,000 FTEs [17]. Workers in the commercial fishing industry have the highest occupational fatality rate in the United States, nearly 35 times higher in 2011 than the rate for all United States workers [18]. More specifically in the United States, the average annual fishing related fatal injury rate in Alaska was 4.2 per 1,000 person-years from 1980 to 1984 [19]. For 1991-1998 there was a statistically significant decreasing trend, with rates reduced to 1.16 per 1,000 person-years [20,21]. The average annual fatality rate was 1.3 per 1,000 fishermen in 2008. By contrast, the average annual occupational fatality rate among all United States workers during the same period was 0.04 deaths per 1,000 workers $[17,22]$.

\section{CAUSAL FACTORS}

Norway has kept a high priority for safety in fishing with good safety culture, but also the composition of the fishing fleet with larger vessels accounts for the significant decrease in fatal accidents $[7,8]$. While the deep sea fishing vessels ( $L o a)>28 \mathrm{~m}$ represent the highest number of fishermen-years, they also represent the lowest risk. Altogether, this contributes significantly to the average low fatal risk.

On Iceland half of the fatalities were due to foundering, and other frequent causes were falls overboard and falls into the harbour [6].

The vessel disasters in Denmark accounted for more than half of the fatalities and were mainly caused by foundering/capsizing due to stability changes in rough weather and collisions. About one third of fatal accidents occurred on the deck while fishing and the remaining $12 \%$ were due to hazardous embarking/disembarking conditions, due to darkness in foreign ports and alcohol intoxication [12].
In the Polish small-scale fishing industry the fatal injury rate was significantly higher in boats $<13 \mathrm{~m}$ in length. In $96 \%$ of cases, the fatalities were caused by external, non-medical causes, while $60 \%$ were related to sea catastrophes, often involving multiple vessels [15]. Alcohol was implicated in $45 \%$ of deaths where an autopsy was carried out. The study concludes that the mortality rates vary significantly by type of vessel, and alcohol may be a significant contributing causal risk factor.

The fatalities in the United States most commonly was caused by drowning as a result of vessels sinking $(51 \%)$ and falls overboard (30\%). Another $10 \%$ of fatalities ( 51 deaths) were caused by injuries sustained on board vessels, such as entanglement in machinery [17]. The remaining 37 (7\%) deaths occurred while diving or in an event on shore. Among vessel disaster incidents, $28 \%$ were initiated by flooding, $18 \%$ by vessel instability, and $18 \%$ by being struck by a large wave. Severe weather conditions contributed to $61 \%$ of the 148 fatal vessel disasters. Among the crewmembers that died from falling overboard, none of them were wearing a personal flotation device. Of the falls overboard with known causes, 43 (33\%) were caused by trips or slips, $34(26 \%)$ by losing balance, and 21 (16\%) by gear entanglement. In addition, the majority of persons (82\%) who died when they fell overboard were alone on the deck [17].

\section{DISCUSSION}

\section{INCIDENCE RATES AND TRENDS}

This is the first review based on the trends of the incidence rates of fatal injuries in commercial fishing. Except for the United Kingdom, there is an overall decrease of the rates over the last decades. Even if the fatal injury rates cannot precisely be compared due to different methods for calculation of the rates, still the trends and levels can be compared with meaning. As mentioned by several of the authors, the definition of the population of fishermen at risk vary among the countries whether we have data about the full time and/or part-time fishermen. This hinders for a direct and precise comparison of the incidence rates. However, as the same inclusion criteria are kept over the decades this allows for valid national estimates of the national trends and valid comparisons of the difference in the trends among the countries. Even if the incidence rates among the countries are incomparable directly, it is supposed that the differences are limited so that it gives meaning to compare the development of the rates. The following examples illustrate some of the specific problems: The Norwegian incidence rates are calculated per "man-labour years", which is based on the effective number of days at sea while other countries use "man-years" which is the number of active registered professional fishermen. A recalculation by using the "man- 
-years" in the denominator would decrease the Norwegian rates from about 0.8 to 0.56 per 1,000 fishermen per year $[7,8]$. Similarly the United States and Alaska rates are based on FTE man-years that will add bias to an international comparison of the rates. For example if 1,000 fishermen work 9 months full-time per year in fishing they contribute with 750 FTE man-years. The calculated incidence rate based on 750 FTE instead of 1,000 man-years will be increased by a factor 1.3 and give bias to an international comparison.

Another type of comparison problem is seen for Iceland where the fishing fleet is mainly composed of large fishing vessels and merchant seafarers are also included in the risk populations. As they are supposed to have a lower overall injury risk, the total population at risk is incomparable with the other fishing populations.

Concerning the problems to get accurate numbers of active fishermen, The Danish statistics include the leisure time fishermen in the number of all active fishermen, while the Ministry of Agriculture and Fishing only include the full-time fishermen and the incidence rate would be the highest by using the data from the Ministry. The former would give more than 3,000 fishermen-years while the other gives about 2,000 and so obviously this gives very different calculated risks.

There are also problems to get the comparable "nominator" data, which is the number of dead fishermen by accidents. For example fishermen working alone on the vessel, fall overboard and never get found give rise to inaccuracy, in this case the results is a lower number of fatal cases. Altogether even as the data are to some degree incomparable it gives meaning to compare the rates by taking the size of the bias in consideration. Canada and Norway are supposed to have the lowest risks. And even if the data to some degree are incomparable we believe that the difference in safety is a reality.

\section{CAUSAL FACTORS}

Fatigue has been shown to be a risk factor in line with alcohol intoxication in the merchant fleet and it was also recently reported to be a significant causal risk factor in fishing [23]. Of 81 fishermen, $60 \%$ stated that their personal safety had been at risk at work because of fatigue, and $75 \%$ reported increasing fatigue effects with length of period at sea. Thus, fatigue seems to be an important factor to be addressed in future preventive efforts $[21,24]$. In a survey of fatigue in British fishermen, $60 \%(n=48)$ believed their personal safety had been at risk because of fatigue at work, $16 \%(n=13)$ had been involved in a fatigue related accident, and $44 \%(n=36)$ said they had worked to the point of exhaustion or collapse. These findings suggest a problem that requires further investigation [25]. An overall prevention model of fatigue comes from the Cardiff research group in the advice for seafaring [23]. While half of the fatalities in fishing are related to vessel disasters, the impact of fatigue in fishing vessel disasters has not yet been accounted for. There are no studies to test the hypothesis that fatigue can be an important causal risk factor for navigation errors in fishing. Thus such studies are strongly needed to learn how to prevent fatigue as a risk factor for fishing vessel disasters.

\section{IMPACT OF THE PREVENTION PROGRAMS}

In Norway a multifactorial risk prevention model has been developed and implemented for many years and includes: manning, sleep quality, noise, training of the crew, regular risk assessment, safety culture, etc. Control of alcohol consumption in harbour is also relevant [7]. Safety training for apprentices and for all fishermen has been obligatory in the Nordic countries for many years. The programs seem to have had very good impacts, as Norway has the lowest rate and a significant decrease of the rates over the decades, even if the modernisation of the fishing fleet has probably also a significant impact.

On Iceland, the Maritime Safety and Survival Training Centre was launched in 1985 and initially provided safety courses as a voluntary option for fishermen [6]. The training programs have subsequently been made mandatory for all fishermen on vessels over 20 Gross Registered Tons and also here we see a significant impact with reduced fatal rates.

The Danish Fishermen's Occupational Health Services was founded in 1995 and from then on the safety and health programs were developed and implemented for all the fishermen [26]. A special focus has been put on work place risk assessments on board and the prevention of disasters for small vessels especially concerning improved vessel stability and the rescue fleet. Regular and repeated safety training for all fishermen and improved safety measures are implemented. Further, regular inspections by the Maritime Authorities have now become obligatory for all fishing vessels, including the vessels below $20 \mathrm{~m}$ length overall. Similar to the other countries, there has been a significant decrease of the fatal accident rates most probably as an effect of the prevention programs [12].

In the United Kingdom, there has been extensively safety regulations especially after the United Kingdom entered in the European Community. Reasons for the lack of reduction over time in the fatal accident rate include increases in fatal accidents among lone fishermen, increases for other high risk groups such as crews of potting vessels, increased financial pressures in the fishing industry and increases in accidents from unseaworthy, unstable and badly maintained fishing vessels, and a lack of use of personal flotation devices [27]. 
In Canada since 1992 the Transportation Safety Board has made a long row of recommendations concerning fishing safety, though the various provincial legislative regimes differ with some taking more comprehensive approach than others. Many of the recommendations have been acted on and it seems to have had an overall very positive impact with a stable low fatal incidence rate of about 0.20-0.30, the lowest seen in fishing [14].

The federal Commercial Fishing Industry Vessel Safety Act of 1988 resulted in this safety improvement in Alaska. These specific regulations were tailored to prevent drowning associated with vessels capsizing and sinking in Alaska's commercial fishing industry by keeping fishermen warm and afloat until rescue $[20,21]$.

As around half of the fatalities are related to fall overboard with subsequent risk of hypothermia and drowning, the prevention of hypothermia has been of high priority in the prevention programs. SINTEF (Selskapet for INdustriell og TEknisk Forskning ved norges tekniske hoegskole - The Foundation for Scientific and Industrial Research at the Norwegian Institute of Technology) in Norway and the Danish Fishermen's Occupational Health Services, Denmark, have both independently developed some effective rescue measures based on the same philosophy: they should be multifaceted to prevent fall overboard and hypothermia, give improved vessels stability and better rescue float measures for small fishing vessels. The latest development is an international project based in Norway with development of a protective clothing concept which is now widely in use in Norway and other countries. The concept combines good buoyancy, thermal protection, tear and puncture resistance, head and hand protection, emergency warning, and positioning systems $[28,29]$.

\section{CONCLUSIONS}

On average, the fatal incidence rates in fishing decreased by around 50\% from 1980 to 2010 to a stable level of 0.25-1.2 per 1,000 man-years; still smaller vessels have the highest risk.

The relative risk for fatal injuries in fishing, compared with other industries in the countries, varied from a factor 25 to 50 and even up to 120 times higher for the United Kingdom fishing.

Approximately $50 \%$ of fatal injuries in fishing in all the countries are related to vessel disasters and drowning, therefore the prevention of vessels disasters and prevention of hypothermia are extremely important with expected large human and economic impact.

Fatigue and lack of good safety climate have been mentioned in different countries as important causal factors that need to be prevented in order to minimise the vessels disasters and so the number of deaths.
The implemented safety programs over the decades seem to have had significant impacts on the fatal injury risk with variations in the countries and the size of the vessels.

\section{REFERENCES}

1. European Occupational Safety and Health Risk Assessment [Internet]. [cited 2014 Feb 26].

2. Ramazzini B. Diseases of workers: De Morbis Artificum, New York Academy of Medicine, The History of Medicine Series, 23, Hafner; 1964.

3. Schilling RS. Trawler fishing: an extreme occupation. Proc R Soc Med 1966; 59: 405-410.

4. Conway GA. Casting their lot upon the water: commercial fishing safety. Lancet 2002; 360: 503-504.

5. Roberts SE, Williams JC. Update of mortality for workers in the UK merchant shipping and fishing sectors. Risk 2005; 1976: 58.

6. Petursdottir G, Hjoervar T, Snorrason H. Fatal accidents in the Icelandic fishing fleet 1980-2005. Int Marit Health 2007; 58: 47-58.

7. Aasjord HL. Tools for improving safety management in the Norwegian Fishing Fleet occupational accidents analysis period of 1998-2006. Int Marit Health 2006; 57: 76-84.

8. McGuinness E, Aasjord HL, Utne IB, Holmen IM. Fatalities in the Norwegian fishing fleet 1990-2011. Saf Sci 2013; 57: 335-351.

9. Rafnsson V, Gunnarsdóttir H. Mortality among Icelandic seamen. Int J Epidemiol 1994; 23: 730-736.

10. Vanggaard L, Neilsen S. [Working environment in Danish fisheries. 1. Occupational accidents]. Ugeskr Laeger 1977; 139: 413-419.

11. Jensen OC. Analyse af anmeldte fiskeriulykker til Søfartsstyrelsen, 1988-1992 [Analysis of the reported fishing injuries to the Danish Maritime Authority 1988-1992]. Esbj Inst Marit Med South Jutl Univ Cent 1994; 1: 80.

12. Laursen LH, Hansen HL, Jensen OC. Fatal occupational accidents in Danish fishing vessels 1989-2005. Int J Inj Contr Saf Promot 2008; 15: 109-117.

13. Danish Maritime Authority, Accidents in fishing 2010; 1: 80.

14. Marine Investigation Report Safety Issues Investigation into Fishing Safety in Canada Report Number M09z0001 2012. Minister of Public Works and Government Services Canada 2012.

15. Jaremin B, Kotulak E. Mortality in the Polish small-scale fishing industry. Occup Med Oxf Engl 2004; 54: 258-260.

16. Hasselback $P$, Neutel $\mathrm{Cl}$. Risk for commercial fishing deaths in Canadian Atlantic provinces. Br J Ind Med 1990; 47: 498-501.

17. Lincoln JM, Lucas DL. Occupational fatalities in the United States commercial fishing industry, 2000-2009. J Agromedicine 2010; 15: 343-350.

18. Centers for Disease Control and Prevention (CDC). Commercial fishing deaths: United States, 2000-2009. MMWR Morb Mortal Wkly Rep 2010; 59: 842-845.

19. Schnitzer PG, Landen DD, Russell JC. Occupational injury deaths in Alaska's fishing industry, 1980 through 1988. Am J Public Health 1993; 83: 685-688.

20. Lincoln J, Husberg B, Conway G. Improving safety in the Alaskan commercial fishing industry. Int J Circumpolar Health 2001; 60: 705-713.

21. Thomas TK, Lincoln JM, Husberg BJ, Conway GA. Is it safe on deck? Fatal and non-fatal workplace injuries among Alaskan commercial fishermen. Am J Ind Med 2001; 40: 693-702.

22. Conway GA, Lincoln JM, Husberg BJ, Manwaring JC, Bensyl DM, Choromanski DM. Alaska's model program for occupational injury prevention: applying surveillance for effective public health practice. Int J Circumpolar Health 2001; 60: 714-723. 
23. Smith A, Allen P, Wadsworth E. Seafarer fatigue: the Cardiff research programme [Internet]. Centre for Occupational and Health Psychology, Cardiff University UK 2006 [cited 2014 Mar 12]. http://www. dieselduck.net/library/.

24. Gander P, van den Berg M, Signal L. Sleep and sleepiness of fishermen on rotating schedules. Chronobiol Int 2008; 25: 389-398.

25. Allen P, Wellens B, Smith A. Fatigue in British fishermen. Int Marit Health 2010; 62: 154-158.

26. The Danish Fishermen's Occupational Health Services [Internet]. [cited 2014 May 31]. Available from: http://www.f-a.dk/.
27. Morgan R. Exploring how fishermen respond to the challenges facing the fishing industry: a study of diversification and multiple-job holding in the English Channel fishery. PhD Thesis. University of Portsmouth 2013.

28. Geving IH, Reitan J, Sandsund M, Faerevik H, Reinertsen RE, Aasjord H. Safer work clothing for fishermen. Int Marit Health 2006; 57: 94-102.

29. Storholmen TCB, Naesgaard OP, Faerevik H, Reitan J, Holmen IM, Reinertsen RE. Design for end-user acceptance: requirements for work clothing for fishermen in Mediterranean and northern fishing grounds. Int Marit Health 2012; 63: 32-39. 